\title{
A head-to-head Phase III study comparing zanubrutinib versus ibrutinib in patients with Waldenström macroglobulinemia
}

\author{
Constantine S Tam*,1,2,3,4, Veronique LeBlond ${ }^{5}$, William Novotny ${ }^{6}$, Roger G Owen ${ }^{7}$, \\ Alessandra Tedeschi ${ }^{8}$, Siminder Atwal ${ }^{6}$, Aileen Cohen ${ }^{6}$, Jane Huang ${ }^{6}$ \& Christian Buske ${ }^{9}$ \\ ${ }^{1}$ Peter MacCallum Cancer Center, Melbourne, Victoria, Australia \\ ${ }^{2}$ University of Melbourne, Parkville, Victoria, Australia \\ ${ }^{3}$ St Vincent's Hospital, Fitzroy, Victoria, Australia \\ ${ }^{4}$ Royal Melbourne Hospital, Parkville, Victoria, Australia \\ ${ }^{5}$ UPMC GRC 11, Pitié Salpêtrière Hospital, Paris, France \\ ${ }^{6}$ BeiGene Company Ltd, San Mateo, CA 94403, USA \\ ${ }^{7}$ St James' University Hospital, Leeds, UK \\ ${ }^{8}$ Niguarda Cancer Center, Milan, Italy \\ ${ }^{9} \mathrm{CCC}$ Ulm, University Hospital Ulm, Ulm, Germany \\ *Author for correspondence: Tel.: +61 385595 000; Fax: +61 385597 739; constantine.tam@petermac.org
}

Waldenström macroglobulinemia (WM), an incurable B-cell malignancy, is sensitive to Bruton tyrosine kinase (BTK) inhibition with ibrutinib, a first-generation BTK inhibitor. Off-target effects of ibrutinib against TEC- and EGFR-family kinases are implicated in some adverse events. Patients with CXCR4 ${ }^{W H I M}$ and MYD88 ${ }^{L 265 P}$ mutations or who are MYD88 ${ }^{W T}$ have less sensitivity to ibrutinib than those with MYD88 ${ }^{2265 P}$ and CXCR4 ${ }^{W T}$ disease. Zanubrutinib, a next-generation BTK inhibitor with potent preclinical activity in WM and minimal off-target effects, showed sustained BTK occupancy in peripheral blood mononuclear cells from patients with B-cell malignancies and promising responses in advanced WM. Described here is a head-to-head Phase III study comparing efficacy and safety of zanubrutinib and ibrutinib in WM patients. Effect of MYD88 and CXCR4 mutation status will be assessed.

First draft submitted: 28 February 2018; Accepted for publication: 18 April 2018; Published online: 5 June 2018

Keywords: Bruton tyrosine kinase $\bullet$ BTK inhibitor • clinical trial • Waldenström macroglobulinemia $\bullet$ zanubrutinib

Introduction to the trial

Here we describe an ongoing, head-to-head Phase III study of zanubrutinib (BGB-3111) versus ibrutinib in patients with $M Y D 88^{M U T}$ Waldenström macroglobulinemia (WM; including an exploratory analysis of zanubrutinib in patients with $M Y D 88^{W T}$ ), assessing the rate of complete response (CR) or very good partial response (VGPR) based on independent review, according to an adaptation of the Sixth International Workshop on WM (IWWM) criteria (Clinicaltrials.gov identifier NCT03053440).

\section{Background \& rationale}

Waldenström macroglobulinemia

WM is a rare lymphoproliferative disorder characterized by bone marrow (BM) infiltration with monoclonal IgMsecreting lymphoplasmacytic cells [1]. Incidence of WM is estimated at 3.8 cases per million and increases with advancing age [2,3]. Median age at diagnosis is 73 years, with Caucasians and men at higher risk for WM than other races and women, respectively [3]. Approximately 1000-1500 new patients are diagnosed in the USA each year [4].

Hemoglobin levels, BM infiltration, serum monoclonal spike and $\beta_{2}$-microglobulin levels are significant predictors of need for therapy [2]. Physical manifestations of the disorder include hepatomegaly, splenomegaly and lymphadenopathy, with fatigue related to normocytic anemia [2]. Per the IWWM-7 consensus criteria, indication for treatment may be either clinically or laboratory-based, with therapeutic strategy determined by individual 
patient and disease characteristics, including age, comorbidities, renal function and disease phenotype, as well as eligibility for stem cell transplant and other complications [5].

More than $90 \%$ of WM patients have a recurrent somatic activating mutation of MYD $88^{L 265 P}$ that triggers downstream IL-1 receptor-associated kinase-mediated NF- $\kappa$ B signaling and supports WM cell survival [6,7], whereas the WHIM-like mutation of CXCR $4^{\text {WHIM }}$ (in approximately $30 \%$ of patients) is associated with tumor progression and drug resistance [8,9]. Patients with both $M Y D 88^{L 265 P}$ and $C X C R 4^{W H I M}$ mutations have significantly higher $\mathrm{BM}$ disease involvement, higher serum IgM levels and symptomatic disease that requires therapy [10,11]. However, CXCR4 mutation status does not appear to affect mortality rates, at least in the context of conventional rituximabcontaining therapies. Patients with $M Y D 88^{W T}$, however, have significantly higher risk for death than those with MYD88 ${ }^{L 265 P}[10,11]$.

\section{Current therapies for WM}

Until the recent approval of ibrutinib for WM, chemotherapy in combination with rituximab were the predominant choices for first-line therapy [2]. There is no consensus on a standard of care for the treatment of newly diagnosed WM patients. The current Mayo Clinic-preferred regimen for newly diagnosed WM is rituximab plus bendamustine or dexamethasone, rituximab and cyclophosphamide when disease burden is low [12]. Likewise, the National Comprehensive Cancer Network (NCCN) Guidelines for WM and lymphoplasmacytic lymphoma lists rituximab-based therapies and ibrutinib monotherapy among several regimens for first- and later-line therapies [13]. Proteasome inhibitor-based therapies are emerging as a treatment option for WM. Carfilzomib, a neuropathysparing proteasome inhibitor, is currently indicated in the USA for off-label treatment of WM, and the NCCN lists carfilzomib-based regimens as treatment options for this disease [14]. Despite generally favorable responses with current therapies, WM is incurable, and relapse is almost inevitable [15].

\section{Bruton tyrosine kinase inhibition as a therapeutic strategy for WM}

Bruton tyrosine kinase (BTK), a critical signaling component of the B-cell receptor pathway, is constitutively activated in WM and a key mediator in tumor cell survival $[7,11,16]$. Inhibition of BTK has emerged as a promising strategy for targeting B-cell malignancies, including WM, particularly in patients harboring the MYD $88^{L 265 P}$ mutation [17]. Ibrutinib is a first-generation BTK inhibitor approved by the US FDA for use in any line of therapy for WM and by the EMA for the treatment of WM in adult patients who have received at least one prior therapy, or in first line treatment for patients unsuitable for chemoimmunotherapy [18,19].

In clinical studies of previously treated WM, ibrutinib demonstrated an overall response rate (ORR) of 90.5\%, a major response rate (MRR) of 73\% (including 16\% VGPR), 2-year progression-free survival (PFS) and overall survival (OS) rates of 69.1 and $95.2 \%$, respectively, and a 3-year event-free survival rate of $68 \%$ [20,21]. In rituximab-refractory WM, ORR was $90 \%$, and MRR was $71 \%$; estimated 18 -month PFS and OS were 86 and $97 \%$, respectively [22]. The most common adverse events (AEs) reported with ibrutinib were neutropenia and thrombocytopenia, with diarrhea, bleeding, hypertension and atrial fibrillation as common nonhematologic toxicities [21,23].

Analyses of ibrutinib-treated patients by CXCR4/MYD88 mutation status have shown an association of CXCR4 ${ }^{W H I M} M Y D 88^{L 265 P}$ mutations with relative resistance to ibrutinib, manifested by lower response rates, slower response kinetics and shorter PFS [9]. Response rates and survival outcomes are poor in MYD88 ${ }^{W T}$ patients [9]. The NCCN consequently updated their recommendation for ibrutinib in WM, noting the lower ORR and absence of major responses with ibrutinib in patients with $M Y D 88^{W T}$ [13]. In addition to the association of mutational status with resistance to therapy, ibrutinib has several off-target effects, including inhibition of other tyrosine kinases, such as EGFR, ITK, TEC and HCK, an important survival determinant in WM that is activated by mutated MYD88 [24]. These effects have been implicated in the development of bleeding, rash, diarrhea and atrial fibrillation in ibrutinib-treated patients [24,25]. As such, other BTK inhibitors with more specificity for BTK are currently being explored and developed [25].

\section{Zanubrutinib}

Zanubrutinib is a next-generation small-molecule oral BTK inhibitor that, like ibrutinib, forms an irreversible covalent bond at Cys481 in the adenosine triphosphate-binding pocket of the BTK active site. It is a potent BTK inhibitor with greater selectivity for BTK than ibrutinib, with minimal off-target inhibition of TEC- and EGFR-family kinases in in vitro cell-based assays [26,27]. In Phase I testing, drug exposure in zanubrutinib-treated 


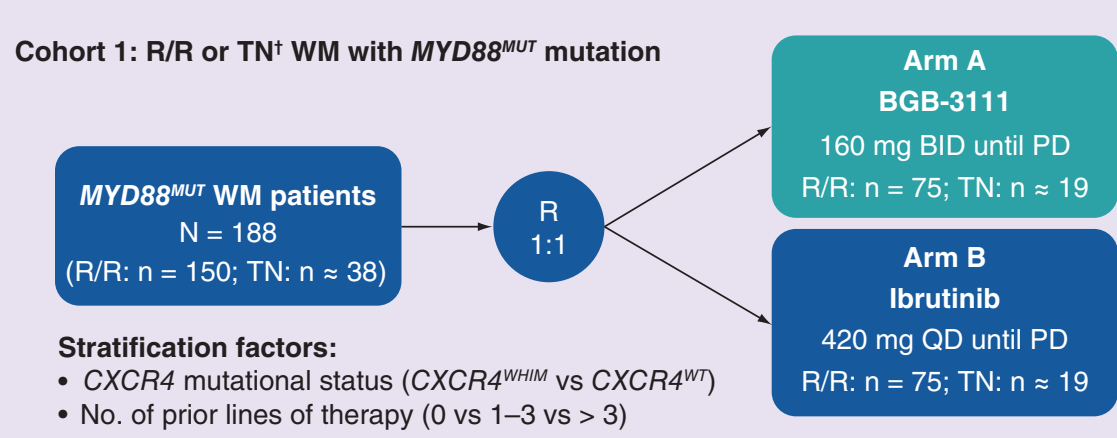

Cohort 2: WM with wild-type MYD88; present in $\approx 10 \%$ of enrolled patients

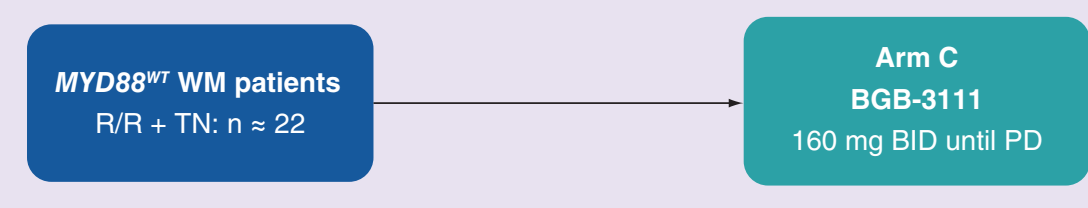

Figure 1. Study design.

†TN must be unsuitable for standard chemoimmunotherapy.

BID: Twice daily; PD: Progressive disease; PO: Orally; QD: Once daily; R: Randomization; R/R: Relapsed/refractory; TN: Treatment-naive; WM: Waldenström macroglobulinemia.

patients was approximately eight-times higher than those reported for ibrutinib-treated patients [27,28]. Complete and continuous BTK occupancy by zanubrutinib has been observed in peripheral blood mononuclear cells, as well as in nodal lymphocytes of patients with advanced B-cell malignancies [27].

Initial results from an ongoing first in human Phase I trial (AU-003) presented at the 14th International Conference on Malignant Lymphoma demonstrated encouraging clinical activity for zanubrutinib in 42 patients with WM (33 with relapsed/refractory disease and nine treatment-naive), with an ORR of $90 \%$ and an MRR of $76 \%$ (including $43 \%$ VGPR) [29]. All four patients with both the CXCR $4^{W H I M}$ and MYD8 $8265 P$ mutations responded to zanubrutinib, with one patient having a VGPR. Of the five patients who had MYD $88^{W T}$ disease, four responded to zanubrutinib, including one VGPR. Response depth improved over time, and no patients had progressed after a median of 12.3 months of treatment. Preliminary safety data did not show any unanticipated safety signals based on the known profile of BTK inhibition in WM. The recommended Phase II dose was established at $160 \mathrm{mg}$ twice daily [29]. The high VGPR rate suggested that high drug exposure and inhibition of BTK in tissue sites may improve therapeutic efficacy in WM.

\section{Design}

This Phase III, randomized, open-label, multicenter study will compare the efficacy and safety of zanubrutinib and ibrutinib in patients with WM who require therapy according to the consensus panel criteria from IWWM7 [5]. The primary objective of the study is to demonstrate the superiority of zanubrutinib versus ibrutinib, as measured by the proportion of patients achieving a CR or VGPR. The study population will consist of two cohorts, based on MYD88 sequencing of baseline BM samples at a central laboratory. Cohort 1 will include at least 150 relapsed/refractory patients and approximately 38 treatment-naive patients with $M Y D 88^{M U T}$ (mutations in exon 5 of the MYD88 gene, including the L265P mutation), randomized 1:1 to either zanubrutinib or ibrutinib. Cohort 2 will consist of treatment-naive or relapsed/refractory patients with $M Y D 88^{W T}$ receiving zanubrutinib in a third, nonrandomized arm to evaluate efficacy among patients lacking a known ibrutinib-sensitive MYD88 variant. The study schema is presented in Figure 1. The study opened to accrual in January 2017 and is recruiting patients from approximately 80 sites in the European Union, Asia-Pacific and North America.

Patients will be stratified by CXCR4 mutational status (CXCR $4^{W H I M}$ vs $\left.C X C R 4^{W T}\right)$ and number of prior lines of therapy (0 vs $1-3$ vs $>3)$. 


\section{Key eligibility criteria}

Eligible patients must be aged $\geq 18$ years, be able to provide written informed consent and have a clinical and definitive histologic diagnosis of WM that is either relapsed/refractory disease or treatment-naive. Patients who are treatment-naive must be considered by the treating physician to be unsuitable for standard chemoimmunotherapy regimens based on the patient's comorbidities and risk factors. At least one criterion for treatment according to the IWWM-7 consensus criteria must be met. All patients should have measurable disease, defined by serum IgM level $>0.5 \mathrm{~g} / \mathrm{dl}$, Eastern Cooperative Oncology Group performance status of 0-2, adequate BM function (neutrophils $\geq 0.75 \times 10^{9} / 1$ and platelets $\geq 50 \times 10^{9} / 1$ independent of growth factor support [and transfusion for platelets] within 7 days of study entry), creatinine clearance $\geq 30 \mathrm{ml} / \mathrm{min}$ (by Cockcroft-Gault equation or estimated glomerular filtration rate), AST and ALT $\leq 3 \times$ upper limit of normal and bilirubin $\leq 2 \times$ upper limit of normal. Relapse after stem cell transplant must be $\geq 3$ months for autologous hematopoietic stem cell transplantation and $\geq 6$ months for allogeneic stem cell transplant.

Exclusion criteria include prior exposure to other BTK inhibitors; currently active, clinically significant cardiovascular disease (e.g., uncontrolled arrhythmia, congestive heart failure, any class 3/4 cardiac disease, as defined by the New York Heart Association); QTcF prolongation (>480 ms); major surgery within 4 weeks; central nervous system involvement by WM; and concomitant warfarin, vitamin $\mathrm{K}$ antagonist or medications that are strong cytochrome P450, cytochrome P450 3A inhibitors or inducers.

\section{Study procedures}

Patients will be divided into two cohorts according to results of MYD88 sequencing performed by a central laboratory at screening. Sequencing of $M Y D 88$ will be done using a highly-sensitive modified allele-specific PCR assay (NeoGenomics, CA, USA) followed by Sanger sequencing [30]. Mutational status of CXCR4 will be determined using bidirectional sequencing of the C-terminus to identify mutations associated with WHIM syndrome [31]. Patients with MYD88 $8^{M U T}$ (cohort 1) will be randomized to receive zanubrutinib (arm A) or ibrutinib (arm B). Patients with $M Y D 88^{W T}$ (cohort 2) will be assigned to receive zanubrutinib (arm C). Zanubrutinib will be administered at $160 \mathrm{mg}$ ( $80 \mathrm{mg} \times$ two capsules) by mouth twice daily (with interval of $\geq 8 \mathrm{~h}$ ). Ibrutinib will be administered at $420 \mathrm{mg}$ (140 mg $\times$ three capsules) administered by mouth once daily until disease progression or nontolerable toxicity. Treatment will be administered at approximately the same time each day. Each treatment cycle consists of 28 days.

In the event of hematologic or nonhematologic toxicities suspected to be related to treatment, doses may be held for a maximum of two consecutive cycles. Dose reductions because of toxicities will consist of two steps: zanubrutinib dose may be reduced from the starting dose of $160 \mathrm{mg}$ twice daily to $80 \mathrm{mg}$ twice daily, then $80 \mathrm{mg}$ once daily; ibrutinib dose may be reduced from a starting dose of $420 \mathrm{mg}$ once daily to $280 \mathrm{mg}$ once daily, then $140 \mathrm{mg}$ once daily.

\section{Outcome measures}

Primary and secondary end points apply to cohort 1 only. The primary end point is the proportion of patients achieving either CR or VGPR, as determined by the Independent Review Committee (IRC), using an adaptation of the response criteria updated at the Sixth IWWM [32] and NCCN guidelines [13] for lymphoplasmacytic lymphoma and WM, requiring a decrease in extramedullary disease if present at baseline to achieve a VGPR or PR (Table 1). The primary efficacy analysis will be conducted 12 months after the last patient is randomized. Response assessments will be made at the beginning of every 4-week cycle starting cycle 2 day 1 for the first 48 weeks, then every three cycles thereafter until disease progression.

Secondary efficacy end points include MRR by IRC, defined as the proportion of patients achieving CR, VGPR or PR; duration of response (DOR; as assessed by IRC and by investigator) for CR, VGPR and PR; PFS (by IRC and by investigator); resolution of treatment-precipitating symptoms; and antilymphoma effect. For patients who discontinue for reasons other than disease progression, efficacy evaluations will continue until disease progression.

Secondary safety end points include the incidence, timing and severity of treatment-emergent AEs according to the Common Terminology Criteria for Adverse Events version 4.03, and the incidence of AEs of special interest including grade $\geq 3$ diarrhea, severe bleeding (Common Terminology Criteria for Adverse Events version 4.03 grade $\geq 3$ bleeding of any site or central nervous system bleeding of any grade), any-grade new-onset atrial fibrillation, any-grade pneumonitis and any treatment-emergent AE leading to study drug discontinuation. All 


\begin{tabular}{|c|c|}
\hline Response category & Definition \\
\hline CR & $\begin{array}{l}\text { - Normal serum IgM values } \\
\text { - Disappearance of monoclonal protein by immunofixation } \\
\text { - No histological evidence of bone marrow involvement } \\
\text { - Complete resolution of lymphadenopathy/splenomegaly (if present at baseline) }\end{array}$ \\
\hline VGPR & $\begin{array}{l}\text { - Monoclonal IgM protein is detectable } \\
\text { - } \geq 90 \% \text { reduction in serum IgM level from baseline or normal serum IgM values } \\
\text { - Improvement in extramedullary disease, lymphadenopathy/splenomegaly if present at baseline } \\
\text { - No new signs or symptoms of active disease }\end{array}$ \\
\hline PR & $\begin{array}{l}-\geq 50 \% \text { reduction of serum IgM from baseline } \\
\text { - Reduction in lymphadenopathy/splenomegaly (if present at baseline) }\end{array}$ \\
\hline MR & - At least $25 \%$ but $<50 \%$ reduction of serum IgM from baseline \\
\hline SD & - Not meeting criteria for CR, VGPR, PR, MR or PD \\
\hline PD & $\begin{array}{l}\text { At least one of the following: } \\
\text { - Confirmed } \geq 25 \% \text { increase in serum IgM and total increase of } \geq 500 \mathrm{mg} / \mathrm{dl} \text { from nadir (on treatment) } \\
\text { - New lymph nodes }>1.5 \mathrm{~cm} \text {, or } \geq 50 \% \text { increase from nadir in SPD of }>1 \text { node, or } \geq 50 \% \text { increase in longest diameter } \\
\text { of a previously identified node } \\
\text { - New splenomegaly or } \geq 50 \% \text { increase from nadir in enlargement } \\
\text { - New extranodal disease } \\
\text { - New or recurrent involvement in bone marrow } \\
\text { - New symptomatic disease }\end{array}$ \\
\hline \multicolumn{2}{|c|}{$\begin{array}{l}\text { CR: Complete response; MR: Minor response; PD: Progressive disease; PR: Partial response; SD: Stable disease; SPD: Sum product of diameter; VGPR: Very good partia } \\
\text { response. } \\
\text { Data taken from [32]. }\end{array}$} \\
\hline
\end{tabular}

patients will be followed for AEs until 30 days after the last dose of study drug. All treatment-related AEs and serious AEs will be followed until resolution or stabilization.

Cohort 1 patients will be evaluated for impact of CXCR4 mutation status on MRR, time-to-major response, PFS and OS. Quality of life assessments, based on the European Organisation for Research and Treatment of Cancer QoL Questionnaire-C30 and EuroQoL Group-5D questionnaire, will be administered every 12 weeks for the first 48 weeks, then every 24 weeks thereafter [33]. Exploratory end points for cohort 2 only include efficacy (response rates, OS, PFS and DOR) and safety of zanubrutinib in patients with $M Y D 88^{W T}$ WM. Zanubrutinib pharmacokinetics will also be evaluated in patients receiving zanubrutinib (cohort 1 arm A and cohort 2). For patients who fail to respond or manifest disease relapse, mechanisms of disease resistance will be explored by correlating potential resistance biomarkers from paired biopsy specimens with efficacy outcomes.

\section{Statistics}

All inferential statistics described below refer to efficacy comparisons between treatment arms in cohort 1. Separate descriptive statistics will be used to report efficacy and safety of zanubrutinib in cohort 2 .

\section{Analysis set}

The intention-to-treat population will include all enrolled patients who are assigned to a treatment arm and will be the primary population for analysis of response rate, PFS, DOR and OS. The safety population will include all patients who receive any dose of zanubrutinib or ibrutinib and will be used for all safety analyses. The per-protocol population will include patients who receive any dose of study medication with no major protocol deviations and will be the secondary analysis population for efficacy. The pharmacokinetics population will include all patients for whom valid zanubrutinib pharmacokinetic parameters can be estimated.

\section{Sample size}

Approximately 210 patients will be enrolled: 188 patients with $M Y D 88^{M U T}$ WM (150 relapsed/refractory and 38 treatment-naive) in cohort 1 , randomized 1:1 to either arm A or arm B, and approximately 20-25 MYD88 ${ }^{W T}$ patients (15-20 with relapsed/refractory and five treatment-naive) in cohort 2, assigned to arm C. Patients with no prior therapy will comprise no more than $20 \%$, and those that have relapsed or refractory WM will comprise $80 \%$ or more of the study population. 
Analysis methods

The primary end point of CR and VGPR will be assessed by an IRC based on the best overall response, defined as the best response recorded from the cohort assignment date until either data cut-off date or the start of a new antineoplastic treatment. A Cochran-Mantel-Haenszel test of rate difference will be used to test the hypothesis after adjusting for stratification factors. The primary efficacy analysis will be conducted 12 months after the cohort assignment of the last patient.

The secondary efficacy end point of MRR will be analyzed similarly to the primary analysis. For other secondary and exploratory efficacy end points, PFS, OS and DOR will be estimated by Kaplan-Meier method, using the stratified log-rank test to determine differences between treatment arms, and Cox regression will be used to estimate the hazard ratios. Changes in extramedullary disease will be compared using an analysis of variance model, with treatment arm as the group variable. Percentages of patients with decreased lymphoplasmacytoid lymphocytes or with resolution/reduction of pretreatment lymphadenopathy or hepatosplenomegaly as verified by computed tomographic scan will be compared using the Fisher's Exact test.

\section{Conclusion}

Despite recent progress in understanding the pathophysiology of WM and the development of new therapeutic approaches, WM remains an incurable disease with significant morbidity. Relapse requiring further treatment is inevitable for many patients.

BTK inhibition represents an effective therapeutic strategy for management of WM, with the first-generation BTK inhibitor ibrutinib demonstrating activity and now being approved for all lines of therapy. However, treatmentspecific toxicity and the emergence of ibrutinib resistance have led to the development of next-generation BTK inhibitors, including tirabrutinib (ONO/GS-4059) and acalabrutinib (ACP-196) [25]. Although clinical data in patients with WM are limited, both tirabrutinib and acalabrutinib have demonstrated activity and favorable toxicity profiles in early-phase studies of patients with relapsed/refractory B-cell malignancies [34,35]. The efficacy of acalabrutinib in previously treated WM patients is currently being investigated in a Phase Ib/II study [36]. Zanubrutinib, a next-generation BTK inhibitor designed to have greater potency and improved selectivity and specificity relative to ibrutinib, has the potential to further improve outcomes in patients with WM. This head-tohead Phase III trial is designed both to compare the safety and efficacy of zanubrutinib with those of ibrutinib and to evaluate the effects of CXCR4 and MYD88 mutations on outcomes. Results of the study will help to address the importance of targeted specificity against BTK and the role of off-target effects in the treatment of WM.

\footnotetext{
Financial \& competing interests disclosure

Trial supported by BeiGene. C Tam has received consulting fees and research support from BeiGene and Janssen. $V$ LeBlond has received consulting fees from Abbvie, Roche and Janssen, has participated on Speakers' Bureaus for Abbvie, Janssen, Roche, and Gilead and has had travel expenses paid for by Roche and Abbvie. R Owen has received honoraria from Janssen, Celgene, and Takeda; consultancy fees from Pharmacyclics, Janssen, and Celgene; and meeting/travel support from Janssen and Celgene. A Tedeschi has served on advisory boards for Janssen, Abbvie and Amgen. W Novotny, S Atwal, A Cohen and J Huang are employees of Beigene and hold stock options. C Buske has received research grants from Janssen and Roche and has participated on advisory boards for Roche, Janssen, Celgene, Amgen. The authors have no other relevant affiliations or financial involvement with any organization or entity with a financial interest in or financial conflict with the subject matter or materials discussed in the manuscript apart from those disclosed.

Medical writing and editorial assistance was funded by BeiGene and was provided, under the direction of the authors, by JV Stelten of Bio Connections.
}

Open access

This work is licensed under the Attribution-NonCommercial-NoDerivatives 4.0 Unported License. To view a copy of this license, visit http://creativecommons.org/licenses/by-nc-nd/4.0/ 


\section{Waldenström macroglobulinemia}

- Waldenström macroglobulinemia (WM) is a rare, incurable B-cell malignancy for which the mutation status of MYD88 and CXCR4 is associated with clinical outcomes and response to therapy.

- Since the recent US FDA and EMA approval for ibrutinib for all lines of WM therapy, ibrutinib has become a cornerstone of treatment for patients with WM, beside rituximab-based therapies.

\section{Bruton tyrosine kinase inhibition in WM}

- Bruton tyrosine kinase (BTK) inhibition represents a promising strategy for treatment of B-cell malignancies, with ibrutinib, a first-generation BTK inhibitor, having shown favorable major response rates, progression-free survival and an acceptable safety profile.

- Ibrutinib has several off-target effects, including inhibition of other tyrosine kinases, such as EGFR, ITK, and TEC.

- Inferior outcomes with ibrutinib have been observed in patients with CXCR4 ${ }^{W H I M} M Y D 88^{L 265 P}$ mutations or MYD88 ${ }^{W T}$ versus MYD88 ${ }^{L 265 P}$ CXCR4 ${ }^{W T}$.

\section{Zanubrutinib}

- Zanubrutinib is a highly potent, next-generation small-molecule oral BTK inhibitor that has greater selectivity for BTK than ibrutinib, with minimal off-target inhibition of TEC- and EGFR-family kinases in in vitro cell-based assays.

- Initial results from an ongoing Phase I trial suggested encouraging clinical activity with zanubrutinib in patients with WM, demonstrating an overall response rate of $90 \%$, and a major response rate of $76 \%$.

Phase III trial

- This is a head-to-head Phase III, randomized, open-label, multicenter study comparing efficacy and safety of zanubrutinib to those of ibrutinib in patients with WM.

- Patients enrolled must have WM that is either relapsed/refractory disease or treatment-naive (treatment-naive must be considered unsuitable for standard chemoimmunotherapy regimens).

- The study population will consist of two cohorts: patients with any MYD88 mutation, randomized 1:1 to either zanubrutinib or ibrutinib (cohort 1); and patients with $M Y D 88^{W T}$ receiving zanubrutinib (cohort 2).

- Patients will be stratified by CXCR4 mutational status (CXCR4 ${ }^{W H I M}$ vs $C X C R 4^{W T}$ ) and number of prior lines of therapy (0 vs $1-3$ vs $>3$ ).

- Zanubrutinib will be administered at $160 \mathrm{mg}$ orally twice daily. Ibrutinib will be administered at $420 \mathrm{mg}$ administered orally once daily until progression or nontolerable toxicity.

- Starting on day 1 of cycle 2, response assessments will be made at the beginning of every 4-week cycle for first 48 weeks, then every three cycles thereafter until disease progression.

Objectives

- The primary objective of this study is to compare the efficacy of zanubrutinib versus ibrutinib in patients with MYD88 $8^{M U T}$ WM.

- Secondary objectives are to further compare efficacy, clinical benefit and antilymphoma effects and to evaluate safety and tolerability of zanubrutinib versus ibrutinib in patients with MYD88 ${ }^{M U T}$ WM.

- The primary end point is the proportion of patients in cohort 1 achieving either complete response or very good partial response determined by the Independent Review Committee.

- Secondary end points include adverse events, major response rate, duration of response, progression-free survival, resolution of treatment-precipitating symptoms and antilymphoma effect.

- Exploratory end points include quality of life, safety and efficacy of zanubrutinib in cohort 2 patients (with $M Y D 88^{W T}$ ) and zanubrutinib pharmacokinetics in patients receiving zanubrutinib.

- Cohort 1 patients will be evaluated for impact of CXCR4 mutation status on efficacy end points.

Statistics

- The primary end point of complete response and very good partial response in cohort 1 will be compared using a Cochran-Mantel-Haenszel test of rate difference after adjusting for stratification factors.

- The secondary efficacy end point of major response rate will be analyzed similarly to the primary analysis.

- For other secondary and exploratory efficacy end points, progression-free survival, overall survival and duration of response will be estimated by Kaplan-Meier method, using the stratified log-rank test to determine differences between treatment arms, and Cox regression will be used to estimate the hazard ratios.

Conclusion

- Zanubrutinib is a next-generation BTK inhibitor designed to have greater potency and improved selectivity and specificity than ibrutinib and has potential to improve outcomes and reduce side effects in patients with WM.

- Results of the study will help to address the importance of targeted specificity against BTK and role of off-target effects in treatment of WM. 


\section{References}

Papers of special note have been highlighted as: $\bullet$ of interest; $\bullet \bullet$ of considerable interest

1. Castillo JJ, Garcia-Sanz R, Hatjiharissi E et al. Recommendations for the diagnosis and initial evaluation of patients with Waldenstrom macroglobulinaemia: a task force from the 8th International Workshop on Waldenstrom macroglobulinaemia. Br. J. Haematol. 175(1), 77-86 (2016).

2. Gertz MA. Waldenstrom macroglobulinemia: 2017 update on diagnosis: risk stratification: and management. Am. J. Hematol. 92(2), 209-217 (2017).

3. Wang H, Chen Y, Li F et al. Temporal and geographic variations of Waldenstrom macroglobulinemia incidence: a large population-based study. Cancer 118(15), 3793-3800 (2012).

4. About Waldenstrom Macroglobulinemia: what are the key statistics about Waldenstrom macroglobulinemia? www.cancer.org/cancer/waldenstrom-macroglobulinemia/about/key-statistics.html

5. Dimopoulos MA, Kastritis E, Owen RG et al. Treatment recommendations for patients with Waldenstrom macroglobulinemia (WM) and related disorders: IWWM-7 consensus. Blood 124(9), 1404-1411 (2014).

6. Treon SP, Xu L, Yang G et al. MYD88 L265P somatic mutation in Waldenstrom's macroglobulinemia. N. Engl. J. Med. 367(9), 826-833 (2012).

7. Yang G, Zhou Y, Liu X et al. A mutation in MYD88 (L265P) supports the survival of lymphoplasmacytic cells by activation of Bruton tyrosine kinase in Waldenstrom macroglobulinemia. Blood 122(7), 1222-1232 (2013).

8. Roccaro AM, Sacco A, Jimenez C et al. C1013G/CXCR4 acts as a driver mutation of tumor progression and modulator of drug resistance in lymphoplasmacytic lymphoma. Blood 123(26), 4120-4131 (2014).

9. Treon SP, Meid K, Gustine J et al. Long-term follow-up of previously treated patients who received ibrutinib for symptomatic Waldenstrom's macroglobulinemia: update of pivotal clinical trial. Blood 130(Suppl. 1), 2766 (2017).

10. Treon SP, Gustine J, Xu L et al. MYD88 wild-type Waldenstrom macroglobulinaemia: differential diagnosis: risk of histological transformation: and overall survival. Br. J. Haematol. 180(3), 374-380 (2018).

11. Treon SP, Cao Y, Xu L, Yang G, Liu X, Hunter ZR. Somatic mutations in MYD88 and CXCR4 are determinants of clinical presentation and overall survival in Waldenstrom macroglobulinemia. Blood 123(18), 2791-2796 (2014).

12. Kapoor P, Ansell SM, Fonseca R et al. Diagnosis and management of Waldenstrom macroglobulinemia: Mayo Stratification of Macroglobulinemia and Risk-Adapted Therapy (mSMART) Guidelines 2016. JAMA Oncol. 3(9), 1257-1265 (2017).

13. Clinical Practice Guidelines in Oncology: Waldenström's macroglobulinemia/lymphoplasmacytic lymphoma version 1.2018. www.nccn.org/professionals/physician_gls/pdf/waldenstroms.pdf

14. Leblond V, Kastritis E, Advani R et al. Treatment recommendations from the Eight International Workshop on Waldenstrom's macroglobulinemia. Blood 128(10), 1321-1328 (2016).

15. Oza A, Rajkumar SV. Waldenstrom macroglobulinemia: prognosis and management. Blood Cancer J. 5, e394 (2015).

16. Argyropoulos KV, Vogel R, Ziegler C et al. Clonal B cells in Waldenstrom's macroglobulinemia exhibit functional features of chronic active B-cell receptor signaling. Leukemia 30(5), 1116-1125 (2016).

17. Treon SP, Xu L, Hunter Z. MYD88 mutations and response to ibrutinib in Waldenstrom's macroglobulinemia. N. Engl. J. Med. 373(6), 584-586 (2015).

- A post hoc analysis of pivotal ibrutinib trial in previously treated Waldenstrom macroglobulinemia: showing a lack of major responses in patients with $M Y D 88^{W T}$.

18. Cheson BD, Fisher RI, Barrington SF et al. Recommendations for initial evaluation: staging: and response assessment of Hodgkin and non-Hodgkin lymphoma: the Lugano classification. J. Clin. Oncol. 32(27), 3059-3068 (2014).

19. IMBRUVICA ${ }^{\circledR}$ (2018). www.imbruvica.com/docs/librariesprovider7/default-document-library/prescribing-information.pdf

20. Palomba ML, Bantilan K, Meid K et al. Long-term follow-up of a pivotal Phase II trial of ibrutinib for relapsed Waldenström's macroglobulinemia. IWWM-9(2016). http://wmworkshop.org/images/Amsterdam-2016/Abstracts/Session-9/Palomba-9.pdf

21. Treon SP, Tripsas CK, Meid K et al. Ibrutinib in previously treated Waldenstrom's macroglobulinemia. N. Engl. J. Med. 372(15), 1430-1440 (2015).

- Pivotal trial of ibrutinib in previously treated Waldenstrom macroglobulinemia: demonstrating an overall response rate of $90.5 \%$ and major response rate of $73.0 \%$. Estimated 2-year progression-free survival and overall survival rates were 69.1 and $95.2 \%$, respectively.

22. Dimopoulos MA, Trotman J, Tedeschi A et al. Ibrutinib for patients with rituximab-refractory Waldenstrom's macroglobulinaemia (iNNOVATE), an open-label substudy of an international: multicentre: Phase 3 trial. Lancet Oncol. 18(2), 241-250 (2017).

23. Gustine JN, Meid K, Dubeau TE, Treon SP, Castillo JJ. Atrial fibrillation associated with ibrutinib in Waldenstrom macroglobulinemia. Am. J. Hematol. 91(6), E312-E313 (2016).

24. Yang G, Buhrlage SJ, Tan L et al. HCK is a survival determinant transactivated by mutated MYD88: and a direct target of ibrutinib. Blood 127(25), 3237-3252 (2016). 
25. Wu J, Liu C, Tsui ST, Liu D. Second-generation inhibitors of Bruton tyrosine kinase. J. Hematol. Oncol. 9(1), 80 (2016).

26. Li N, Sun Z, Liu Y et al. BGB-3111 is a novel and highly selective Bruton's tyrosine kinase (BTK) inhibitor. Cancer Res. 75(15 Suppl.), 2597 (2015).

27. Tam C, Grigg AP, Opat S et al. The BTK inhibitor: BGB-3111: is safe: tolerable: and highly active in patients with relapsed/refractory B-cell malignancies: initial report of a Phase I first-in-human trial. Blood 126, 832 (2015).

- Initial results of Phase I trial of zanubrutinib in patients with advanced B-cell malignancies. Overall response rate was $90 \%$, and major response rate was $76 \%$, including $43 \%$ very good partial response. No unanticipated safety signals were reported. Results from this study were used to establish the dose used in the Phase III study.

28. Advani RH, Buggy JJ, Sharman JP et al. Bruton tyrosine kinase inhibitor ibrutinib (PCI-32765) has significant activity in patients with relapsed/refractory B-cell malignancies. J. Clin. Oncol. 31(1), 88-94 (2013).

29. Trotman J, Opat S, Marlton P et al. Bruton's tyrosine kinase (BTK) inhibitor BGB-3111 demonstrates high very good partial response (VGPR) rate in patients with Waldenström macroglobulinemia (WM). Hematol. Oncol. 35(Suppl. 2), 70-71 (2017).

- Abstract presented at the 14th International Conference on Malignant Lymphoma Palazzo dei Congressi in Lugano, Switzerland, 14-17 June 2017. Safety and efficacy of zanubrutinib in an expansion cohort of 46 patients with Waldenström macroglobulinemia were presented. Overall response rate was $93 \%$, and major response rate was $78 \%(39 \%$ very good partial response; $39 \%$ partial response). Of five patients with $M Y D 88^{W T}$ status, there was one very good partial response and one partial response. Zanubrutinib was well tolerated.

30. Albitar A, Ma W, Dedios I, Estella J, Agersborg S, Albitar M. Positive selection and high sensitivity test for MYD88 mutations using locked nucleic acid. Int. J. Lab. Hematol. 38(2), 133-140 (2016).

31. Hunter ZR, Xu L, Yang G et al. Transcriptome sequencing reveals a profile that corresponds to genomic variants in Waldenstrom macroglobulinemia. Blood 128(6), 827-838 (2016).

32. Owen RG, Kyle RA, Stone MJ et al. Response assessment in Waldenstrom macroglobulinaemia: update from the VIth International Workshop. Br. J. Haematol. 160(2), 171-176 (2013).

33. Rabin R, de Charro F, Szende A. EQ-5D Value Sets: Inventory: Comparative Review and User Guide. Springer, Dordrecht, the Netherlands (2007).

34. Walter HS, Rule SA, Dyer MJ et al. A Phase 1 clinical trial of the selective BTK inhibitor ONO/GS-4059 in relapsed and refractory mature B-cell malignancies. Blood 127(4), 411-419 (2016).

35. Byrd JC, Harrington B, O’brien S et al. Acalabrutinib (ACP-196) in relapsed chronic lymphocytic leukemia. N. Engl. J. Med. 374(4), 323-332 (2016).

36. Castillo JJ, Hunter ZR, Yang G, Argyropoulos K, Palomba ML, Treon SP. Future therapeutic options for patients with Waldenstrom macroglobulinemia. Best Pract. Res. Clin. Haematol. 29(2), 206-215 (2016). 
